\title{
Progrès récents du groupe nouveaux lasers de l'IMFM-UM34 CNRS dans le domaine des sources UV-VUV cohérentes et incohérentes pulsées de puissance
}

\author{
B. Fontaine, Ph. Delaporte, M. Sentis et B. Forestier
}

IMFM-UM34 CNRS, Pôle Scientifique de Luminy, case 918, 13288 Marseille Cedex 09, France

\begin{abstract}
Most recent results achieved by The IMFM New Lasers Group on Coherent and incoherent pulsed high power ligth sources are presented. They includes efficient damping of acoustic waves in a high PRF lexcimer laser, high average power high PRF $\mathrm{XeCl}$ Laser achievement, demonstration of high efficiency long pulse, small volume $\mathrm{XeCl}(308 \mathrm{~nm})$ and $\mathrm{KrCl}(222 \mathrm{~nm})$ lasers, obtention of intense long pulses VUV fluorescence emissions from $\mathrm{Xe}^{+} \mathrm{Rb}(164 \mathrm{~nm})$ and $\mathrm{Kr}^{+} \mathrm{Rb}(134 \mathrm{~nm})$, and generation of Intense incoherent VUV emissions induced by a discharge on formed ferrite.
\end{abstract}

Depuis une vingtaine d'années, le Groupe Nouveaux lasers de l'IMFM s'intéresse principalement aux processus physiques pouvant mener à une émission laser intense pulsée répétitive dans le domaine spectral allant du visible à l'ultraviolet lointain et en particulier aux lasers à exciplexes. Cette classe de lasers pulsés ultraviolets à des applications potentielles très importantes dans les domaines de la micro-électronique et de la micro-mécanique et plus généralement dans le traitement des matériaux. Les retombées sont également importantes en Génie biologique et médical. suivants:

Le Groupe consacre actuellement plus particulièreemnt ses efforts aux thèmes

* couplages décharge-écoulement-faisceau laser dans les lasers à exciplexes à taux de répétition élevé,

* nouvelles méthodes d'excitation des lasers à exciplèxes permettant d'accroitre la durée d'impulsion et le rendement et d'obtenir des émissions laser à de nouvelles longueurs longueurs d'onde en régime d'impulsions longues,

* faisabilité d'applications spécifiques des lasers à exciplexes à taux de répétition élevé en régimes d'impulsion courte ou longue dans le domaine de l'automobile et de l'aéronautique (métallurgie, traitement de matériaux à stoechiométrie complexe,...),

* possibilités de réalisation de nouveaux lasers dans le VUV à partir d'exciplexes ioniques gaz rare-alcalin à haute pression et haute température excités par faisceau d'électrons ou décharge pulsée.

Des résultats nouveaux concernant ces thèmes ont été obtenus récemment par le groupe. On peut citer notamment les résultats suivants:

* amortissement très efficace, en un temps inférieur à une milliseconde, au moyen d'amortisseurs acoustiques en feutre de nickel, des gradients de densité créés dạns la cavité laser par les 3 familles d'ondes induites par l'excitation très rapide du milieu actif ',

* obtention d'une puissance laser moyenne de 300 Watts pour un taux de répétition de $1 \mathrm{KHz}$ à la longueur d'onde $\lambda=308 \mathrm{~nm}$ (laser XeCl) (systeme d'études laser $\mathrm{LUX}{ }^{2}$,

* obtention d'une émission laser à $\lambda=308 \mathrm{~nm}$ d'une énergie de $220 \mathrm{mj}$ en $200 \mathrm{~ns}$ avec un rendement de $3,5 \%$ dans un volume utile de $70 \mathrm{~cm} 3$ excité par une double décharge à commutateur magnétique très rapide. II à été montré que l'énergie extraite est sensiblement la mème avec $\mathrm{HCl}$ et $\mathrm{BCl} 3$ comme porteur d'halogène ${ }^{3}$,

* obtention, pour la première fois, d'une émission laser de longue durée à $\lambda=222$ $\mathrm{nm}$ (laser $\mathrm{KrCl}$ ). Une énergie laser de $115 \mathrm{mj}$ en $140 \mathrm{~ns}$ à été obtenue avec un rendement de 
$1 \%$ dans un volume 4 tile de $65 \mathrm{~cm} 3$ excité par une double décharge à commutateur magnétique très rapide ${ }^{4,5}$ (Fig. 1),

* mise en évidence d'émissions VUV intenses entre 134 et $164 \mathrm{~nm}$ des excimères ioniques gaz rare-alcalin $\mathrm{Kr}^{+} \mathrm{Rb}, \mathrm{Kr}^{+} \mathrm{Cs}, \mathrm{Xe}^{+} \mathrm{Rb}$ et $\mathrm{Xe}^{+} \mathrm{Cs}$ lorsque des mélanges $\mathrm{Ne}-\mathrm{Kr}-\mathrm{Rb}$, $\mathrm{Ne}-\mathrm{Kr}-\mathrm{Cs}, \mathrm{Ne}-\mathrm{Xe}-\mathrm{Rb}$ et $\mathrm{Ne}-\mathrm{Xe}-\mathrm{Cs}$ à haute pression et haute température sont excités par un faisceau d'électrons en régime d'impulsions longues $(500 \mathrm{~ns})^{6}$,

* mise en évidence d'une émission VUV intense et de rendement élevé lors d'une décharge pulsée sur ferrite formée ${ }^{7}$.

Ces études qui, pour les lasers à halogénure de gaz rares de grande puissance à taux de répétition élevé, sont entreprises dans le cadre du programme EUREKA EU205 et du GDR 919 CNRS qui lui est associé, vont être poursuivies à l'IMFM à moyen terme avec comme objectifs le développement de lasers UV de très grande puissance moyenne et de nouvelles sources coherentes et incoherentes dans les domaine spectraux VUV-UV-visible.

* Travail effectué avec le soutien de la DRET, du MRT (Programme EUREKA Eurolaser EU205), du CNRS (GDR 919) et de la Région Provence-Alpes-Côte d'Azur.

1. Ph.Delaporte, B. Fontaine, B. Forestier, M. Sentis, J.P. Truong, O. Uteza, D. Zeitoun, D. Tarabelli, à paraitre dans Shock Waves (1992).

2. M. Sentis, Ph. Delaporte, B. Forestier, B. Fontaine, IEEE J. Quant. Electr. 27, 2332 (1991).

3. J.M. Hueber, B. Fontaine, Ph.Delaporte, B. Forestier, M. Sentis, Opt. Com. 85, 237 (1991)

4. B. Fontaine, J.M. Hueber, B. Forestier, M. Sentis, Ph. Delaporte, demande de brevet d'invention français No92/03477 du 17 mars 1992.

5. J.M. Hueber, B. Fontaine, N. Bernard, B. Forestier, M. Sentis, Ph.Delaporte, Technical Note IMFM-UM34 CNRS, NL 1992-1, mars 1992; également article soumis pour publication dans Appl. Phys. Letters.

6. Ph.Delaporte, B. Fontaine, B. Forestier, M. Sentis, Conférence Internationale LASERS' 91 , San Diego, décembre 1991, Proceedings à paraitre.

7. R.C. Sze, M. Sentis, Conférence Internationale LASERS' 91, San Diego, décembre 1991, Proceedings à paraitre (1992).

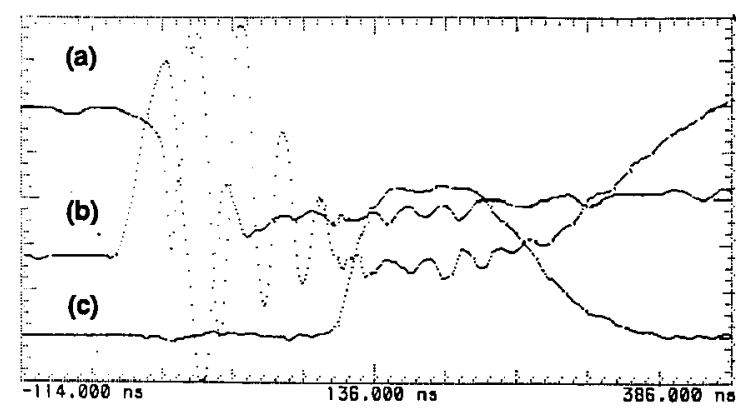

Fig. 1 Laser $\mathrm{KrCl}(\lambda=222 \mathrm{NM})$ en régime d'impulsions longues

Enregistrements oscillographiques types :

* (a) tension aux électrodes (10 KV/ div.)

* (b) courant dans le commutateur magnétique $(5 \mathrm{kA} / \mathrm{div}$.)

* (c) puissance laser (a.u.)

$\mathrm{Ne} / \mathrm{Kr} / \mathrm{XeCl}\left(8000 / 400 / 5 ; \mathrm{P}=3,3 \mathrm{~atm} ., \mathrm{V}_{\mathrm{sp}}=40 \mathrm{KV} ; \mathrm{V}_{\mathrm{PFL}}=11 \mathrm{kV}\right.$
Base de temps: $50 \mathrm{~ns} /$ div.

Energie laser mesurée: $\mathrm{W}_{\mathrm{L}}=115 \mathrm{~mJ}$ 